\title{
Lack of association between NAT2 polymorphism and prostate cancer risk: a meta-analysis and trial sequential analysis
}

\author{
Feng Wang ${ }^{1, *}$, Zhiqiang Qin ${ }^{2, *}$, Shuhui $\mathrm{Si}^{3, *}$, Jingyuan Tang ${ }^{2}$, Lingyan $\mathrm{Xu}^{4}$, Haoxiang $\mathrm{Xu}^{2}$, \\ Ran Li ${ }^{2}$, Peng Han' ${ }^{2}$ and Haiwei Yang ${ }^{1,2}$ \\ ${ }^{1}$ Department of Radiation Oncology, The First Affiliated Hospital of Nanjing Medical University, Nanjing, 210009, China \\ ${ }^{2}$ State Key Laboratory of Reproductive Medicine, Department of Urology, The First Affiliated Hospital of Nanjing Medical \\ University, Nanjing, 210029, China \\ ${ }^{3}$ Research Division of Clinical Pharmacology, The First Affiliated Hospital of Nanjing Medical University, Nanjing, 210009, \\ China \\ ${ }^{4}$ Department of Oncology, The First Affiliated Hospital of Nanjing Medical University, Nanjing, 210009, China \\ *These authors contributed equally to this work \\ Correspondence to: Haiwei Yang, email: haiweiyang@njmu.edu.cn \\ Keywords: $N A T 2 * 4$, gene polymorphism, prostate cancer, meta-analysis
}

Received: March 29, $2017 \quad$ Accepted: June 17, $2017 \quad$ Published: July 05, 2017

Copyright: Wang et al. This is an open-access article distributed under the terms of the Creative Commons Attribution License 3.0 (CC BY 3.0 ), which permits unrestricted use, distribution, and reproduction in any medium, provided the original author and source are credited.

\section{ABSTRACT}

Previous studies have investigated the association between NAT2 polymorphism and the risk of prostate cancer (PCa). However, the findings from these studies remained inconsistent. Hence, we performed a meta-analysis to provide a more reliable conclusion about such associations. In the present meta-analysis, 13 independent case-control studies were included with a total of 14,469 PCa patients and 10,689 controls. All relevant studies published were searched in the databates PubMed, EMBASE, and Web of Science, till March 1st, 2017. We used the pooled odds ratios (ORs) with $95 \%$ confidence intervals (CIs) to evaluate the strength of the association between NAT2*4 allele and susceptibility to PCa. Subgroup analysis was carried out by ethnicity, source of controls and genotyping method. What's more, we also performed trial sequential analysis (TSA) to reduce the risk of type $I$ error and evaluate whether the evidence of the results was firm. Firstly, our results indicated that NAT2*4 allele was not associated with PCa susceptibility $(O R=1.00,95 \% C I=0.95-1.05 ; P=0.100)$. However, after excluding two studies for its heterogeneity and publication bias, no significant relationship was also detected between NAT2* 4 allele and the increased risk of PCa, in fixed-effect model $(O R=0.99$, $95 \% \mathrm{CI}=0.94-1.04 ; P=0.451)$. Meanwhile, no significant increased risk of $\mathrm{PCa}$ was found in the subgroup analyses by ethnicity, source of controls and genotyping method. Moreover, TSA demonstrated that such association was confirmed in the present study. Therefore, this meta-analysis suggested that no significant association between NAT2 polymorphism and the risk of PCa was found.

\section{INTRODUCTION}

Prostate cancer $(\mathrm{PCa})$ is considered as the second most leading cause of cancer-related deaths among men worldwide $[1,2]$. PCa is a multifactorial disease caused by complex interactions between a series of potential risk factors, such as environment, ethnicity, age, and genetic factors. However, the accurate etiology of PCa remained unclear. Nowadays, epidemiologic studies have pointed that several environmental factors such as smoking, ultra-violet radiation, and diet, including meat and fat intake, possibly had an increased risk of developing PCa $[3,4]$. Nevertheless, a large number of candidate genes responsible for PCa have been identified. Genetic factors, particularly single-nucleotide genetic polymorphisms (SNPs), might prove to be reliable in predicting the 
genetic risk of $\mathrm{PCa}$, thus possibly contributing to the primary prevention of this condition [5].

$\mathrm{N}$-acetyltransferase 2 (NAT2) gene is clustered on the short arm of chromosome 8 (8q22) and encodes a phase II xenobioticmetabolizing enzyme [6, 7]. Besides, NAT2 is essentially involved in heterocyclic amines, hydrazines and aromatic metabolites [8, 9]. The alteration of NAT2 acetylator status caused by polymorphosms in NAT2 gene may induce the decrease of enzyme activity and the absence of efficiency in detoxification, thus contributing to increasing cancer susceptibility [10]. There are two major NAT2 phenotypes, including rapid acetylator and slow acetylator. Until now, over sixty NAT2 genetic variants have been discovered in humans, in which NAT2*4 is deemed to the most common allele related to rapid acetylation, and has been designated "wild-type" in history [11-13]. In addition, mutant homozygotes in NAT2 gene are classified as slow acetylator phenotype, while wild-type homozygotes and heterozygotes are categorized into rapid acetylator phenotype.

To date, a number of studies have been performed to elucidate the association between NAT2 genetic polymorphism and susceptibility to $\mathrm{PCa}$. Meanwhile, some studies have demonstrated that people with low NAT2 activity have a higher risk of developing $\mathrm{PCa}$, compared to those with high NAT2 activity [14]. However, the results remained inconsistent or even contrary. Therefore, further assessment is need to be proved to the real association and whether or not rapid acetylation is a risk factor for the development of PCa. Hence, we aimed to conduct a meta-analysis with all accessible case-control studies and trial sequential analysis (TSA) to gain the more precise evidence for the relationship between NAT2 genetic polymorphism and $\mathrm{PCa}$ risk.

\section{RESULTS}

\section{Characteristics of the studies}

Overall, thirteen independent case-control studies were included with a total of $14,469 \mathrm{PCa}$ patients and 10,689 controls in the present meta-analysis [22-34]. Table 1 showed the detailed characteristics and genotype distribution of the selected studies reported the association between NAT $2 * 4$ allele and PCa risk. The process of literature search and selection with specification of reasons was listed in Figure 1. Among these previous studies, ten studies were conducted on Caucasian populations, one was Asians, one was mixed races, and the remaining study was conducted in African population. Furthermore, we consisted of 9 population-based studies and 4 hospitalbased studies, in order to distinguish between different sources of control group. Diverse genotyping methods were included: TaqManSNP (TaqMan), polymerase chain reaction (PCR), PCR-restriction fragment length polymorphism (PCR-RFLP).

\section{Quantitative synthesis results}

Overall, the main results of this meta-analysis about the associations between NAT2 polymorphism and PCa risk were shown in Table 2. Initially, the results from this meta-analysis indicated that there was no significant susceptibility for the NAT2 $* 4$ allele with $\mathrm{PCa}$ risk $(\mathrm{OR}=$ $1.00,95 \% \mathrm{CI}=0.95-1.05 ; P=0.100)$. However, in order to find possible factors, heterogeneity analysis and publication bias were tested. After excluding two studies of Hamasaki et al. and Wang et al. in the existence of heterogeneity and publication bias, the results demonstrated that no significant relationship was also found between $\mathrm{NAT} 2 * 4$ allele and the risk of $\mathrm{PCa}$, in fixed-effects model (OR $=0.99,95 \%$ $\mathrm{CI}=0.94-1.04 ; P=0.451)$ (Figure 2).

In the subgroup analysis by ethnicity, the results were no statistical significance in Caucasian population $(\mathrm{OR}=$ $0.99,95 \% \mathrm{CI}=0.94-1.05$ ) (Figure 3A). Moreover, subgroup analysis by control source groups were also performed, and no statistically significant results were detected in population-based control group $(\mathrm{OR}=0.98,95 \% \mathrm{CI}=0.93-$ $1.03)$ and hospital-based control group $(\mathrm{OR}=1.13,95 \% \mathrm{CI}=$ 0.91-1.41) (Figure 3B). In addition, in the subgroup analysis by different genotyping methods, no significant results of such association were found in TaqMan $(\mathrm{OR}=0.98,95 \%$ $\mathrm{CI}=0.87-1.10), \mathrm{PCR}(\mathrm{OR}=1.13,95 \% \mathrm{CI}=0.93-1.37)$, PCR-RFLP $(\mathrm{OR}=1.02,95 \% \mathrm{CI}=0.83-1.26)$, respectively (Figure 3C). In general, there was no association between NAT2 polymorphism and PCa risk in the comparisons of without NAT2 $* 4$ and with NAT2 $* 4$.

\section{Publication bias}

The Egger's test and the Begg's funnel plot were used to assess the potential publication bias. Before excluding two studies by Hamasaki et al. and Wang et al., the plots exhibited the potential publication bias, because the plots were asymmetric in the Begg's funnel (Figure 4A). Nevertheless, after eliminating this study, the shapes of the funnel plots seemed symmetrically distributed, indicating little evidence of significant publication bias across studies $(P=0.131)$ (Figure 4B).

\section{Test of heterogeneity}

After excluding two studies by Hamasaki et al. and Wang et al., the overall heterogeneity was obviously decreased $(P=0.451)$, which indicated that two studies by Hamasaki et al. and Wang et al. might have generated the origin of heterogeneity (Figure 5). What's more, it was interesting that subgroup analyses were performed to reduce the heterogeneity.

\section{Trial sequential analysis results}

As shown in Figure 6, although the cumulative Z-cure didn't cross the trial sequential monitoring 
Table 1: Characteristics of studies that investigated the association between NAT2 polymorphism and prostate cancer risk

\begin{tabular}{|c|c|c|c|c|c|c|c|c|c|c|c|c|}
\hline Author & Year & Country & Ethnicity & SOC & $\begin{array}{l}\text { Genotyping } \\
\text { methods }\end{array}$ & case & control & $\begin{array}{l}\text { Non-NAT } 2 * 4 \\
\text { of case }\end{array}$ & $\begin{array}{l}\text { Any NAT2 } 24 \\
\text { of case }\end{array}$ & $\begin{array}{c}\text { Non-NAT } 2 * 4 \text { of } \\
\text { control }\end{array}$ & $\begin{array}{c}\text { Any NAT2*4 of } \\
\text { control }\end{array}$ & NOS scores \\
\hline Vilckova & 2014 & Slovak & Caucasian & $\mathrm{HB}$ & PCR-RFLP & 395 & 281 & 172 & 109 & 230 & 165 & 8 \\
\hline Cox & 2011 & NM & Caucasian & PB & NM & 9965 & 6,953 & 4,128 & 2,825 & 5,974 & 3,991 & 9 \\
\hline Kidd & 2011 & USA & African & HB & Taqman & 493 & 190 & 78 & 112 & 201 & 292 & 7 \\
\hline Sharma & 2010 & Mixed & Mixed & PB & Taqman & 2063 & 2,106 & 818 & 1,288 & 814 & 1,249 & 9 \\
\hline Iguchi & 2009 & USA & Caucasian & PB & PCR & 170 & 180 & 111 & 69 & 92 & 78 & 8 \\
\hline Srivastava & 2005 & India & Caucasian & PB & PCR-RFLP & 140 & 130 & 46 & 84 & 62 & 78 & 9 \\
\hline Costa & 2005 & Portugal & Caucasian & PB & PCR-RFLP & 174 & 146 & 60 & 86 & 79 & 95 & 9 \\
\hline Rovito & 2005 & USA & Caucasian & PB & PCR & 146 & 139 & 88 & 51 & 82 & 64 & 8 \\
\hline Gao & 2003 & China & Asian & PB & PCR-RFLP & 112 & 58 & 13 & 45 & 20 & 92 & 9 \\
\hline Hamasaki & 2003 & Japan & Asian & HB & PCR-RFLP & 152 & 111 & 19 & 92 & 13 & 139 & 7 \\
\hline Hein & 2002 & USA & Caucasian & HB & PCR-RFLP & 115 & 47 & 31 & 16 & 60 & 55 & 8 \\
\hline Wang & 2002 & Poland & Caucasian & HB & PCR-RFLP & 17 & 34 & 21 & 13 & 5 & 12 & 8 \\
\hline Wadelius & 1999 & Sweden & Caucasian & PB & PCR & 519 & 331 & 202 & 129 & 320 & 199 & 9 \\
\hline Agundez & 1998 & Spain & Caucasian & PB & PCR & 160 & 94 & 52 & 42 & 83 & 77 & 8 \\
\hline
\end{tabular}

Abbreviations: SOC: Source of controls; PB: Population-based controls; HB: Hospital-based controls; NM: not mentioned; NOS:Newcastle-Ottawa-Scale.

Any NAT2*4:Rapid acetylation; Non-NAT2*4:Slow acetylation.

Notes: The studies of Hamasaki et al and Wang et al (shown in bold) were removed later because of its heterogeneity and publication bias.

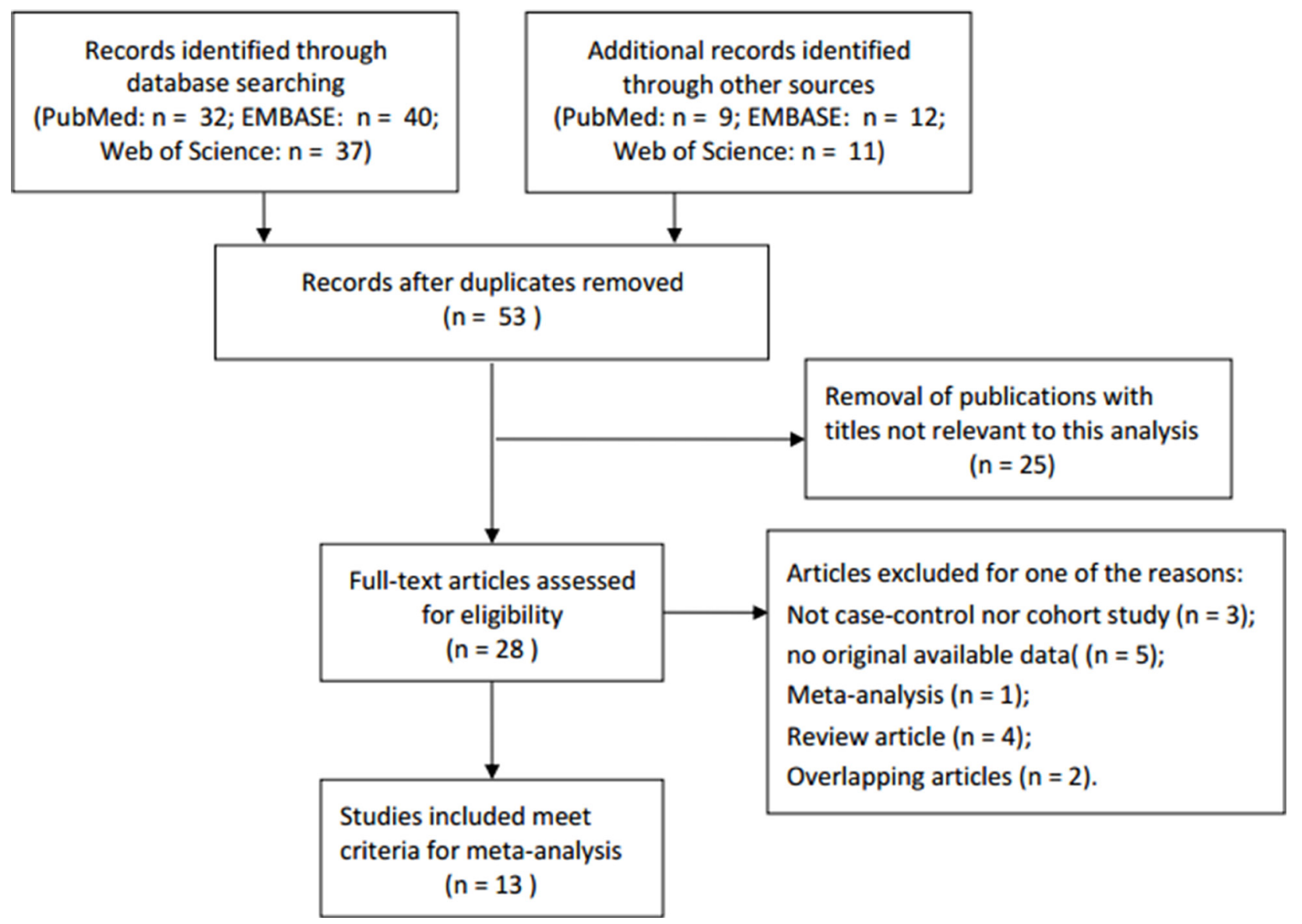

Figure 1: Flow diagram of literature search and selection process. 
Table 2: Meta-analysis results of association between NAT2 polymorphism and prostate cancer risk after the elimination of the two studies by Hamasaki et al and Wang et al

\begin{tabular}{lcccc}
\hline & $\mathbf{N}^{\mathbf{a}}$ & Sample Size & OR (95\% CI)* & $\mathbf{P}^{\mathbf{b}}$ \\
\hline $\begin{array}{l}\text { Total } \\
\text { Ethnicity }\end{array}$ & 12 & 25,107 & $0.99(0.94,1.04)$ & 0.451 \\
$\quad$ Caucasion & & & \\
Genotyping & 9 & 20,085 & $0.99(0.94,1.05)$ & 0.245 \\
$\quad$ PCR-RFLP & 5 & & \\
$\quad$ Taqman & 2 & 1,598 & $1.02(0.83,1.26)$ & 0.169 \\
$\quad$ PCR & 4 & 4,852 & $0.98(0.87,1.10)$ & 0.839 \\
Source of control & & 1,739 & $1.13(0.93,1.37)$ & 0.505 \\
$\quad$ HB & 9 & 1,521 & $1.13(0.91,1.41)$ & 0.371 \\
PB & 3 & 23,586 & $0.98(0.93,1.03)$ & 0.488 \\
\hline
\end{tabular}

${ }^{a}$ Number of studies.

${ }^{\mathrm{b}} P$ value of $Q$ test for heterogeneity.

"Random-effects model was used when $P$ value for heterogeneity test $<0.05$; otherwise, fixed-effects model was used.

boundary, the total number of cases and controls were more than the required information size. To sum up, the sufficient evidence of our results were established and further relevant trials were unnecessary.

\section{DISCUSSION}

The NAT2 gene is located on chromosome 8p21.323.1 and encodes a 290 -amino-acid protein [35, 36]. As an essential phase II enzyme, NAT2 is key in the process of cancer development. Besides, this gene is essential in the metabolism of aromatic heterocyclic amines and hydrazines via $\mathrm{N}$ - and O-acetylation [37]. Alterations to the NAT2 acetylator status caused by variations in the NAT2 gene have been reported to reduce enzymatic activity, resulting in inefficient detoxification and thus leading to increased cancer susceptibility. It has been reported that the variant alleles in NAT2 lead to slow elimination of carcinogenic amines. Two major NAT2 phenotypes have been classified, such as rapid and slow. Several NAT2 genetic variants have been identified in human being, of which NAT2*4 is regarded as the most common allele associated to rapid acetylation. The rapid acetylator phenotype was in association with increased risk of colon, bladder, and $\mathrm{PCa}$ [38-40]. Whereas slow acetylator phenotype was reported to the decreased risk of colon cancer and increased the risk of bladder cancer $[41,42]$.
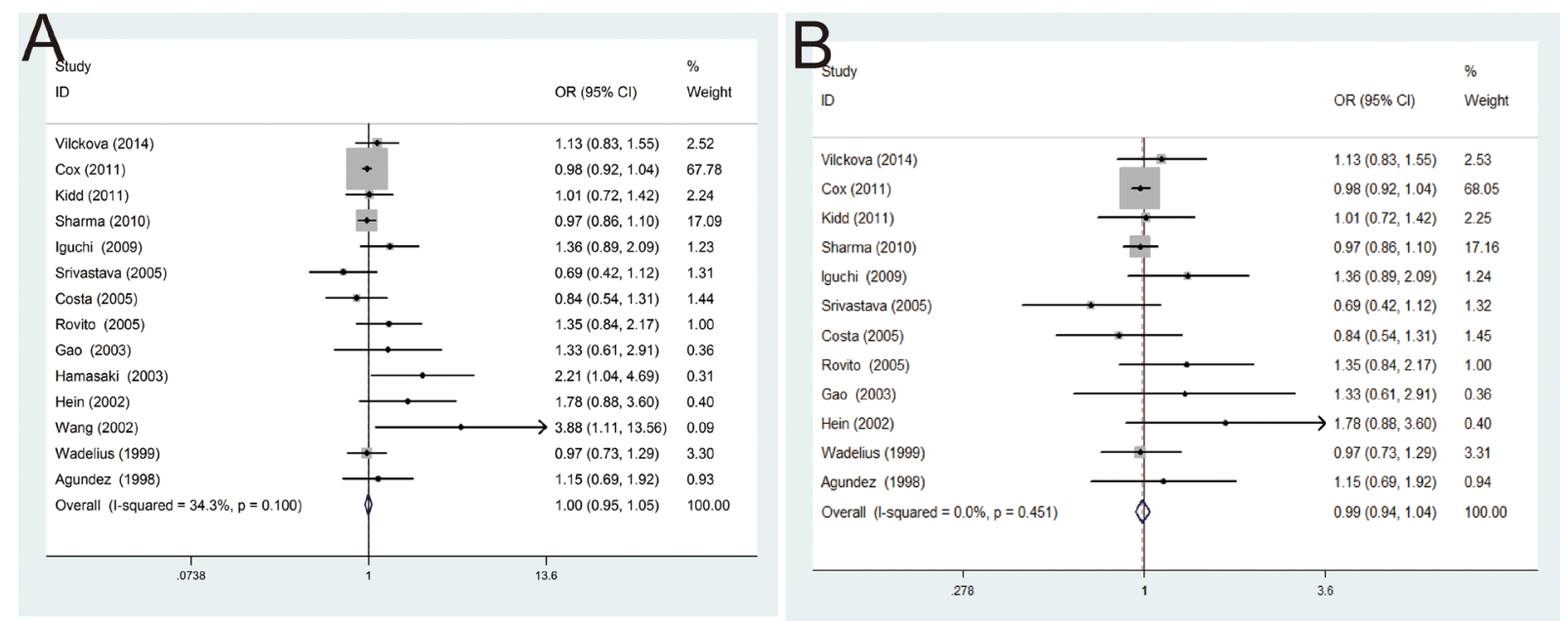

Figure 2: (A) Forest plots of the association between NAT2 polymorphism and PCa susceptibility in fixed model; (B) Forest plots of the association between NAT2 polymorphism and PCa susceptibility in fixed model after omitting two studies by Hamasaki et al. and Wang et al. with heterogeneity and publication bias. 


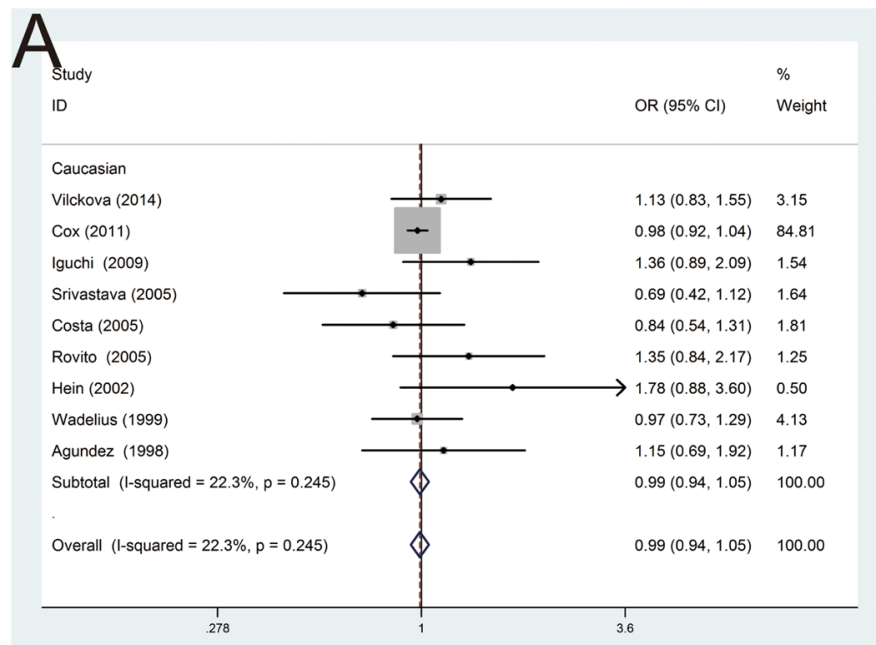

B

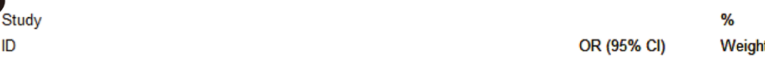

$\mathrm{HB}$ Vilckova (2014) Kidd (2011) Hein (2002)

Subtotal (I-squared $=0.0 \%, p=0.371)$

$\mathrm{PB}$

Cox (2011)

Sharma (2010)

Iguchi (2009)

Srivastava (2005)

Costa (2005)

Rovito (2005)

Gao (2003)

Wadelius (1999)

Agundez (1998)

Subtotal (1-squared $=0.0 \%, p=0.488$ )

Overall (l-squared $=0.0 \%, p=0.451$ )

\begin{tabular}{l|l} 
& \\
\hline 1278 & 1
\end{tabular}

$1.13(0.83,1.55) \quad 2.53$

$1.01(0.72,1.42) \quad 2.25$

$1.78(0.88,3.60) \quad 0.40$

$\begin{array}{rr}1.78(0.88,3.60) & 0.40 \\ 1.13(0.91,1.41) & 5.18\end{array}$

$0.98(0.92,1.04) \quad 68.05$

$0.97(0.86,1.10) \quad 17.16$

$1.36(0.89,2.09) \quad 1.24$

$0.69(0.42,1.12) \quad 1.32$

$0.84(0.54,1.31) \quad 1.45$

$1.35(0.84,2.17) \quad 1.00$

$\begin{array}{ll}1.33(0.61,2.91) & 0.36\end{array}$

$0.97(0.73,1.29) \quad 3.31$

$1.15(0.69,1.92) \quad 0.94$

$0.98(0.93,1.03) \quad 94.82$

$99(0.94,1.04) \quad 100.00$

3.6

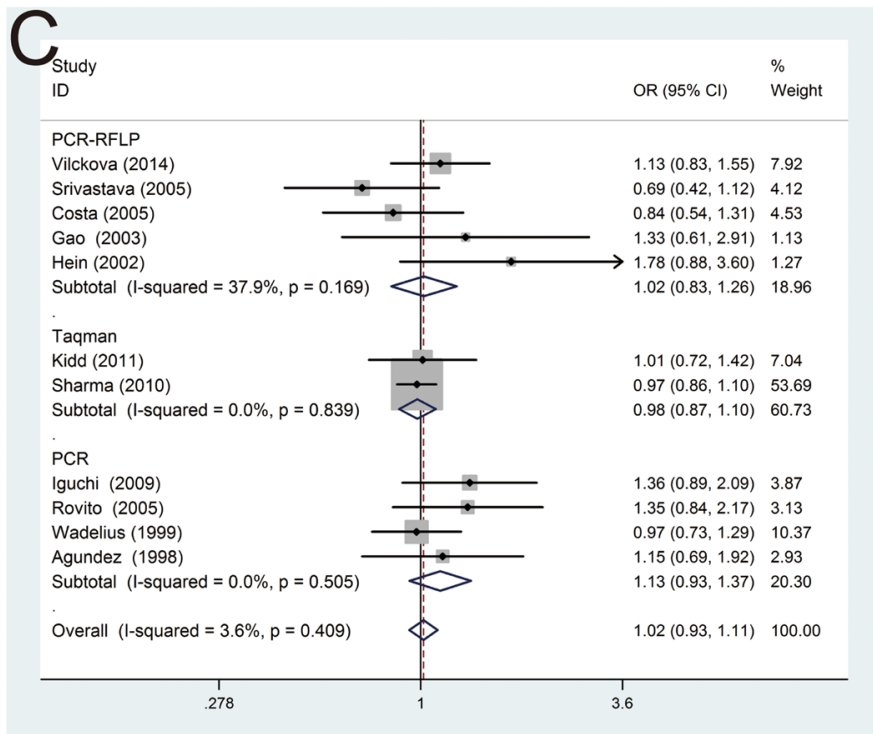

Figure 3: Forest plots of subgroup analysis of the association between NAT2 polymorphism and PCa susceptibility in fixed model. (A) stratified by ethnicity; (B) stratified by source of controls; (C) stratified by genotyping methods. 
A small number of studies have investigated the involvement of NAT2 gene in the etiology of PCa, but they have ultimately led to conflicting results. Therefore, no firm conclusion has been provided regarding NAT2 gene role in PCa risk. On the one hand, the finding by Hamasaki et al. showed that NAT2 slow acetylator genotype increased the risk of PCa in Japanese patients, especially among smokers [14]. On the other hand, the result of another study by Costa $\mathrm{S}$ et al. suggested that NAT2*6E could be associated with the susceptibility of PCa [29]. What's more, another study by Srivastava et al. suggested no significant association between NAT2 genotype and PCa risk was found in the North Indian

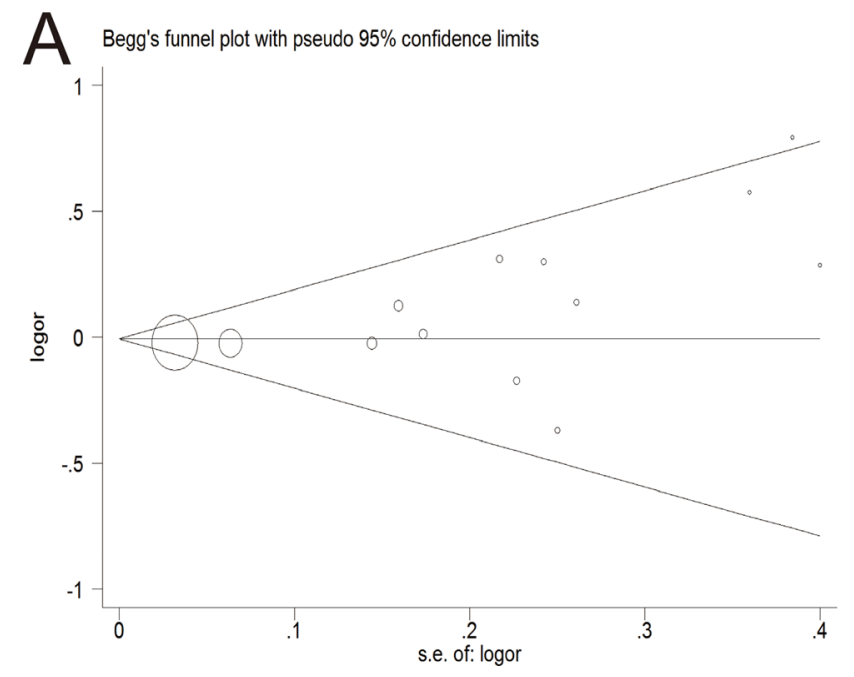

population, but this study reported an association between NAT2 rapid acetylator genotypes and tobacco users with PCa [28]. Hence, no unified conclusions have been provided regarding the role of NAT2 gene polymorphism in PCa risk. In our meta-analysis, we attempt to clarify whether or not the NAT2 polymorphism is correlated with the susceptibility of PCa. Besides, TSA was performed to effectively reduce the risk of type I error and assess whether the evidence of our results was reliable.

Meta-analysis is a powerful tool, which can make the conclusion more credible compared with a single study, especially in analyzing unexplained associations [43]. As the development of the current

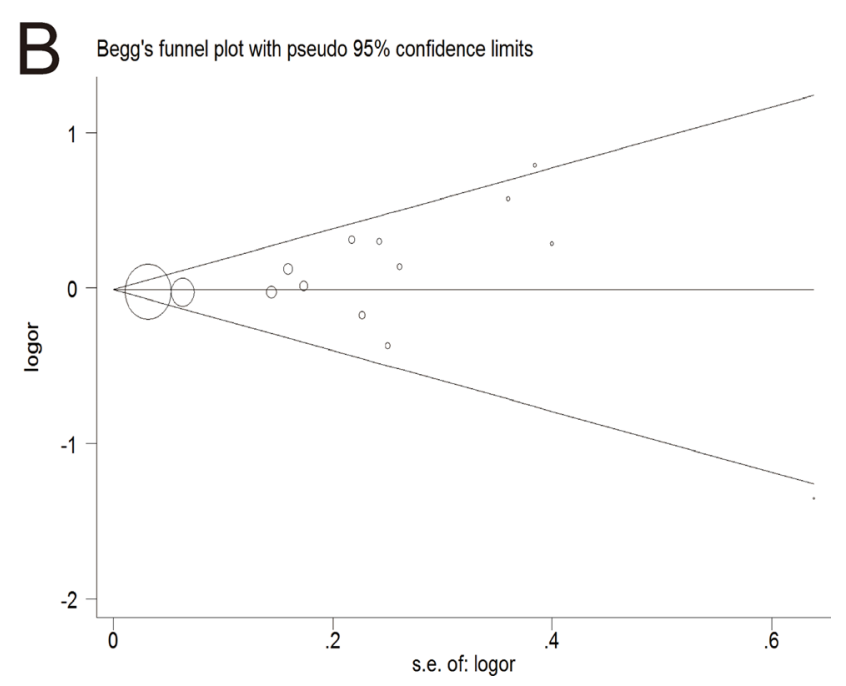

Figure 4: Begg's funnel plot of publication bias test. (A) Before omitting a study of Hamasaki et al. (B) After the exclusion of the study.
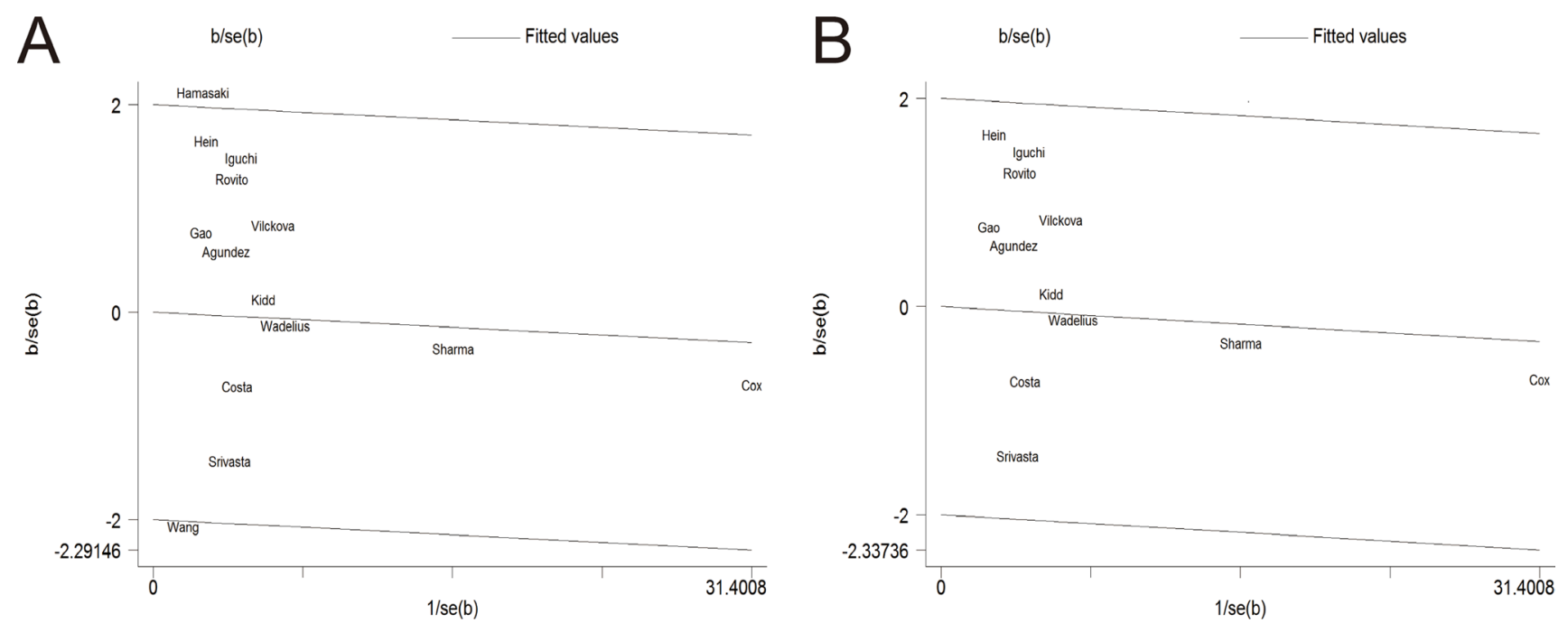

Figure 5: Galbraith plot of the association between NAT2 polymorphism and PCa susceptibility in fixed model. (A) Before removing a study by Hamasaki et al. (B) After the exclusion of the study. 
meta-analysis, we performed it to provide the more comprehensive understanding of the relationship between NAT $2 * 4$ allele and the risk of PCa by different subgroup analysis. As a consequence, we took advantage of metaanalysis to explain this possible association. In the present meta-analysis, 13 independent case-control studies were included with a total of 14,469 PCa patients and 10,689 controls. Our results revealed that no significant relationship was detected between NAT2 $* 4$ allele and the increased risk of $\mathrm{PCa}$. This contradictory could be caused by several factors, including the differences in sample size, genotyping method, study design, and statistical method and so on.

Several subgroup meta-analyses were performed according to different ethnic groups, different source of controls and different methodologies. In racial subgroups, there was no association between NAT2*4 allele and the risk of PCa appeared in Caucasians. Nevertheless, the result might not be very conclusive, because of the relatively small number of African-derived and Asianderived populations used in the meta-analysis. What's more, because of a mixture population from different geographic regions and other races, there was a significant between-study heterogeneity in Caucasians, which might lead to the negative results of our analysis.

In the subgroup analysis by source of controls, no statistical significance about such association was also observed in both population-based control group and hospital-based control group. Besides, there were healthy population and other disease patients with the exception of PCa in the controls included. It was likely that different individuals in the control group might have different risk of developing PCa, thus to affect the quality of the studies.

After stratified analysis by genotyping method, no statistically significant increased PCa risk was shown in TaqMan, PCR, PCR-RFLP and so on. Different genotyping methods had their own benefits in various aspects, which might result in different statistical results. Thus, applying the same appropriate genotyping method might make the meta-analysis results more significance and reliable. What' more, it is necessary to have a unified admission criteria and a larger sample size of the relevant researches.

As an useful tool, TSA is similar to interim analyses in a single trial, where monitoring boundaries are used to decide whether additional trials are needed to evaluate for evidence when a $P$ value is sufficiently small to show the anticipated effect or for futility $[44,45]$. As is well-known to all, traditional meta-analysis may result in type I and type II errors. However, TSA is performed to reduce the risk of type I error by estimation of required information size with an adjusted threshold for statistical significance, and estimate whether further trials are necessary. If the cumulative Z-curve crosses the trial sequential monitoring boundary or the required information size, it shows firm evidence for such study. If not, it is necessary to do an additional clinical trial to reach a consistent conclusion $[19,20,21]$. As shown in our study, the cumulative

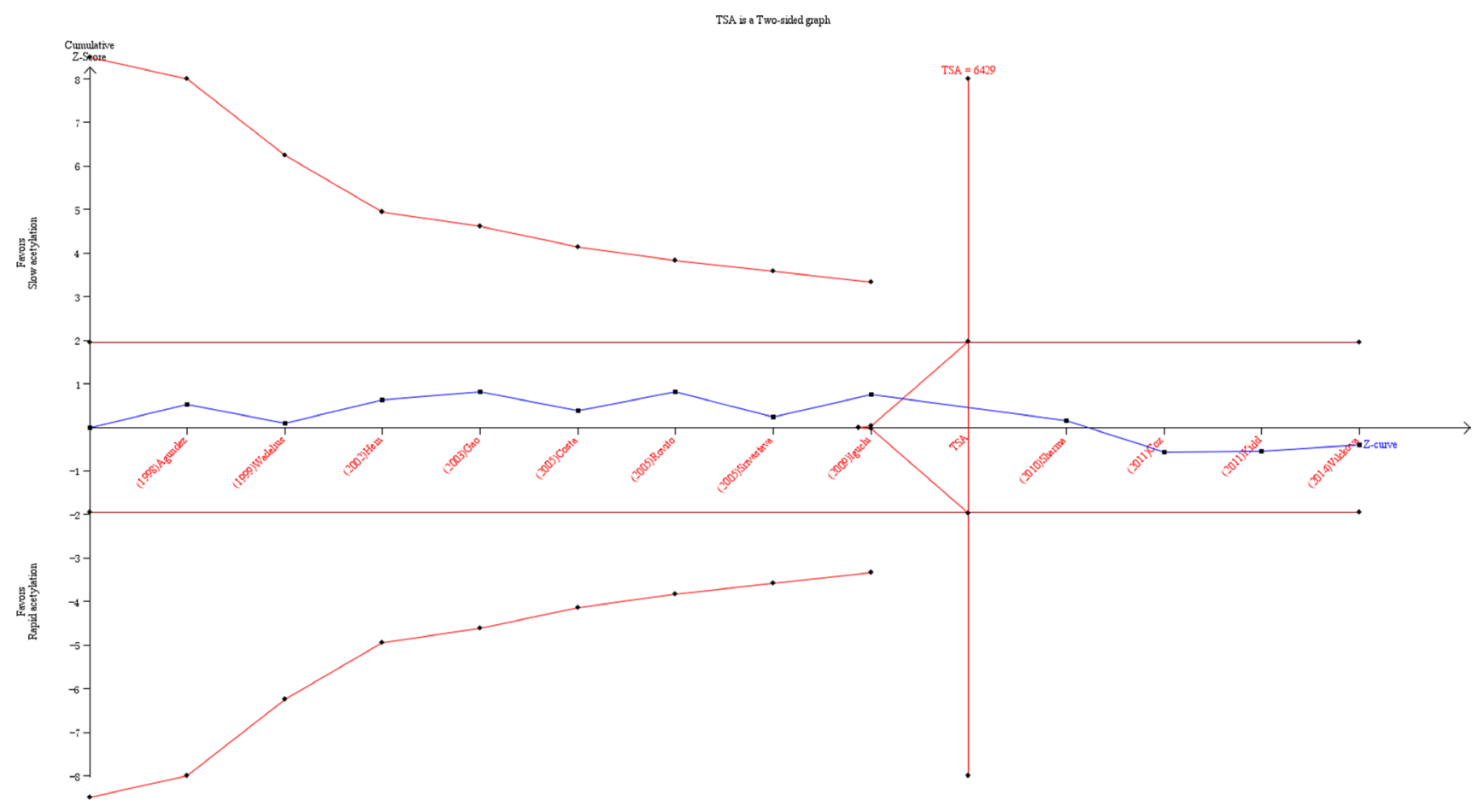

Figure 6: Trial sequential analysis of the association between NAT2 polymorphism and the risk of PCa. The required information size was calculated based on a two side $\alpha=5 \%, \beta=15 \%$ (power $85 \%$ ), and a relative risk reduction of $20 \%$. 
Z-curve has reached the perpendicular line (required information size). Therefore, the evidence of the result is sufficient to reach a conclusion.

Notably, we included more studies with the large sample size to estimate a slight association by this metaanalysis, and this is the first TSA to comprehensively illustrate the impact of NAT2 polymorphism in response to $\mathrm{PCa}$ risk. However, several limitations should be taken into consideration. Firstly, the sample size in each stratified analyses was relatively small and might potentially limit the enough statistical power to explore the real relationship. Therefore, further studies with a larger sample size were still needed to be further validated. Secondly, the pathogenesis of $\mathrm{PCa}$, as a multi-factorial disease, is closely related to environmental backgrounds as well as the interaction with various genetic factors instead of the influence of any single gene. Therefore, gene-to-environment interactions have been an important role to evaluate genetic polymorphisms. Further studies and more original data are needed to evaluate potential gene-to-gene and gene-to-environment interactions. In addition, we couldn't get useful data about the association between NAT2 polymorphism and the risk of PCa in the GWAS database. Although we could not obtain useful data in the GWAS database, a large number of articles related to NAT2*4 allele with PCa risk were found. What's more, a certain deviation may be resulted by a combined analysis of researched population in different ages, ethnicities and types of PCa. Thereby, this risk factor may cause a certain heterogeneity. Additionally, the incidence of $\mathrm{PCa}$ was different among different races. The majority studies included were investigated in Caucasian population in this meta-analysis. Therefore, the outcome of ethnic sub-group analysis might be affected.

\section{MATERIALS AND METHODS}

\section{Literature search}

We searched the relevant studies by electronic database PubMed, EMBASE and Web of Science, with the last search update on March 1st, 2017. The combination of the following terms were used: "N-acetyltransferase-2" or "NAT2", "single nucleotide polymorphism" or "variants", or "polymorphism", and "prostate cancer" or "prostate neoplasm" or "prostate tumor". In addition, we also screened by a manual search from the references of the original articles and retrieved articles considered eligible for the meta-analysis. Moreover, only the latest or more comprehensive study was selected in this meta-analysis, if studies had partly overlapping or familiar subjects.

\section{Inclusion and exclusion criteria}

Eligible studies were selected according to the following criteria: (a) An independent case-control design; (b) The relationship between NAT2 gene polymorphism and susceptibility to PCa was evaluated; (c) The abundant data to evaluate the pooled odds ratios (ORs) with 95\% confidence intervals (CIs) was provided.

Exclusion criteria from this meta-analysis were as follows: (a) Non-case-control studies; (b) Review articles; (c) No original available data of genotype frequency to evaluate this association; (d) Previous duplicated publications.

\section{Data extraction}

Two investigators (Wang $F$ and Qin ZQ) independently extracted the data from the identified studies. In addition, any disagreement was resolved by a discussion with a third reviewer until consensus was based on the main point of view. The following information were recorded in a standardized form: first author's last name, year of publication, country, ethnicity, source of controls (population-based or hospital-based), genotying method, number of cases and controls, and genotype frequency of NAT2 gene polymorphism between cases and controls, respectively.

\section{Quality assessment}

The quality of the studies was assessed using the validated Newcastle-Ottawa Scale (NOS) for nonrandomized studies, including case-control and cohort studies. A study can be awarded a maximum of one star for each point within the selection and exposure categories, and a maximum of two stars can be given for comparability. We considered studies with scores of more than 7 as high-quality studies and only high-quality studies were included in our meta-analysis.

\section{Statistical analysis}

The pooled ORs with $95 \%$ CIs were calculated to assess the strength of association between NAT2 $* 4$ allele and PCa risk. Two models were used in the meta-analysis, including the fixed-effects model (a Mantel-Haenszel method) and the random-effects model (a DerSimonianLaird method). If $P$ values $<0.05$, the random-effects model would be conducted; Otherwise, the fixed effects model would be used to perform meta-analysis. What's more, subgroup analysis was further carried out to explore the potential sources of heterogeneity according to ethnicity, source of controls and genotyping methods. Besides, the influence of publication bias between the studies was estimated by Begg's funnel plots and Egger's linear regression test, and $P<0.05$ was considered to be statistically significant. All above statistical data were conducted by Stata software (version 12.0, Stata Corporation, College Station, TX). 


\section{Trial sequential analysis}

A cumulative meta-analyses with addition of new publishing trials might result in type I and type II errors, because it could increase the risk of random error with repeated significance testing and sparse data [15-17]. Therefore, TSA was introduced to reduce the risk of type I error, which could estimate the required information size with an adjusted threshold for statistical significance $[18,19]$. In the current meta-analysis, TSA was performed by anticipating a $20 \%$ relative risk reduction for efficacy outcome, an overall $5 \%$ risk a type I error and a statistical test power of $80 \%$, to estimate the required diversityadjusted information size [20]. When the cumulative $\mathrm{Z}$-curve crosses the trial sequential monitoring boundary, a sufficient level of evidence has been reached and further studies are unneeded. If the $\mathrm{Z}$ curve dose not cross any of the boundaries and the required information size has not been reached, it is needed to try an additional clinical trial to reach a sufficient conclusion [21]. In this study, we applied the trial sequential analysis software (TSA, version 0.9; Copenhagen Trial Unit, Copenhagen, Denmark, 2011).

\section{CONCLUSIONS}

The results of this meta-analysis demonstrated that no evidence supporting the relationship between NAT2 polymorphism and PCa risk was detected. More importantly, further studies in other ethnic groups, such as Asians, Africans, are needed to give more comprehensive understanding of such association.

\section{CONFLICTS OF INTEREST}

We declare that we have no conflicts of interest.

\section{REFERENCES}

1. Jang TL, Yossepowitch O, Bianco FJ, Scardino PT. Low risk prostate cancer in men under age 65: the case for definitive treatment. Urol Oncol. 2007; 25:510-514.

2. Ondrusova M, Ondrus D, Karabinos J, Muzik J, Kliment J, Gulis G. Trends in prostate cancer incidence and mortality before and after the introduction of PSA testing in the Slovak and Czech Republics. Tumori. 2011; 97:149-155.

3. Sivonova MK, Dobrota D, Dusenka R, Waczulikova I, Slezak P, Matakova T, Mahmoodova S, Mistuna D, Kliment J. Effect of CYP17 and PSA gene polymorphisms on prostate cancer risk and circulating PSA levels in the Slovak population. Mol Biol Rep. 2012; 39:7871-7880.

4. Stamatiou KN. Elderly and prostate cancer screening. Urol J. 2011; 8:83-87.

5. Sivonova MK, Dobrota D, Matakova T, Dusenka R, Grobarcikova S, Habala V, Salagovic J, Tajtakova M,
Pidanicova A, Valansky L, Lachvacs L, Kliment JJ, Nagy V, Kliment J. Microsomal epoxide hydrolase polymorphisms, cigarette smoking and prostate cancer risk in the Slovak population. Neoplasma. 2012; 59:79-84.

6. Blum M, Grant DM, McBride W, Heim M, Meyer UA. Human arylamine $\mathrm{N}$-acetyltransferase genes: isolation, chromosomal localization, and functional expression. DNA Cell Biol. 1990; 9:193-203.

7. Hickman D, Pope J, Patil SD, Fakis G, Smelt V, Stanley LA, Payton M, Unadkat JD, Sim E. Expression of arylamine N-acetyltransferase in human intestine. Gut. 1998; 42:402-409.

8. Hein DW, Doll MA, Gray K, Rustan TD, Ferguson RJ. Metabolic activation of N-hydroxy-2-aminofluorene and N-hydroxy-2-acetylaminofluorene by monomorphic $\mathrm{N}$-acetyltransferase (NAT1) and polymorphic $\mathrm{N}$-acetyltransferase (NAT2) in colon cytosols of Syrian hamsters congenic at the NAT2 locus. Cancer Res. 1993; 53:509-514.

9. De Stefani E, Boffetta P, Mendilaharsu M, Carzoglio J, Deneo-Pellegrini H. Dietary nitrosamines, heterocyclic amines, and risk of gastric cancer: a case-control study in Uruguay. Nutr Cancer. 1998; 30:158-162.

10. Wikman H, Thiel S, Jager B, Schmezer P, Spiegelhalder B, Edler L, Dienemann H, Kayser K, Schulz V, Drings P, Bartsch H, Risch A. Relevance of N-acetyltransferase 1 and 2 (NAT1, NAT2) genetic polymorphisms in non-small cell lung cancer susceptibility. Pharmacogenetics. 2001; 11:157-168.

11. Bolt HM, Selinski S, Dannappel D, Blaszkewicz M, Golka K. Re-investigation of the concordance of human NAT2 phenotypes and genotypes. Arch Toxicol. 2005; 79:196-200.

12. Hein DW, Doll MA. Accuracy of various human NAT2 SNP genotyping panels to infer rapid, intermediate and slow acetylator phenotypes. Pharmacogenomics. 2012; 13:31-41.

13. Taja-Chayeb L, Gonzalez-Fierro A, Miguez-Munoz C, Trejo-Becerril C, Cruz-Hernandez EL, Cantu D, Agundez JA, Vidal-Millan S, Gutierrez O, DuenasGonzalez A. Acetylator status and $\mathrm{N}$-acetyltransferase 2 gene polymorphisms; phenotype-genotype correlation with the sulfamethazine test. Pharmacogenet Genomics. 2011; 21:894-901.

14. Hamasaki $\mathrm{T}$, Inatomi $\mathrm{H}$, Katoh $\mathrm{T}$, Aono $\mathrm{H}$, Ikuyama $\mathrm{T}$, Muratani T, Matsumoto T. N-acetyltransferase-2 gene polymorphism as a possible biomarker for prostate cancer in Japanese men. Int J Urol. 2003; 10:167-173.

15. Turner RM, Bird SM, Higgins JP. The impact of study size on meta-analyses: examination of underpowered studies in Cochrane reviews. Plos One. 2013; 8:e59202.

16. Brok J, Thorlund K, Wetterslev J, Gluud C. Apparently conclusive meta-analyses may be inconclusive--Trial sequential analysis adjustment of random error risk due to repetitive testing of accumulating data in apparently 
conclusive neonatal meta-analyses. Int J Epidemiol. 2009; 38:287-298.

17. Wetterslev J, Thorlund K, Brok J, Gluud C. Trial sequential analysis may establish when firm evidence is reached in cumulative meta-analysis. J Clin Epidemiol. 2008; 61:64-75.

18. Higgins JP, Whitehead A, Simmonds M. Sequential methods for random-effects meta-analysis. Stat Med. 2011; 30:903-921.

19. Thorlund K, Imberger G, Walsh M, Chu R, Gluud C, Wetterslev J, Guyatt G, Devereaux PJ, Thabane L. The number of patients and events required to limit the risk of overestimation of intervention effects in meta-analysis - a simulation study. PLoS One. 2011; 6:e25491.

20. Wetterslev J, Thorlund K, Brok J, Gluud C. Estimating required information size by quantifying diversity in random-effects model meta-analyses. BMC Med Res Methodol. 2009; 9:86.

21. Holst LB, Petersen MW, Haase N, Perner A, Wetterslev J. Restrictive versus liberal transfusion strategy for red blood cell transfusion: systematic review of randomised trials with meta-analysis and trial sequential analysis. BMJ. 2015; 350:h1354.

22. Wang CY, Jones RF, Debiec-Rychter M, Soos G, Haas GP. Correlation of the genotypes for $\mathrm{N}$-acetyltransferases 1 and 2 with double bladder and prostate cancers in a case-comparison study. Anticancer Res. 2002; 22:3529-3535.

23. Vilckova M, Jurecekova J, Dobrota D, Habalova V, Klimcakova L, Waczulikova I, Slezak P, Kliment J, Sivonova MK. Variation in N-acetyltransferase 2 (NAT2), smoking and risk of prostate cancer in the Slovak population. Med Oncol. 2014; 31:987.

24. Cox DG, Dostal L, Hunter DJ, Le Marchand L, Hoover R, Ziegler RG, Thun MJ. N-acetyltransferase 2 polymorphisms, tobacco smoking, and breast cancer risk in the breast and prostate cancer cohort consortium. Am J Epidemiol. 2011; 174:1316-1322.

25. Kidd LC, Vancleave TT, Doll MA, Srivastava DS, Thacker B, Komolafe O, Pihur V, Brock GN, Hein DW. No association between variant $\mathrm{N}$-acetyltransferase genes, cigarette smoking and Prostate Cancer susceptibility among men of African descent. Biomark Cancer. 2011; 2011:1-13.

26. Sharma S, Cao X, Wilkens LR, Yamamoto J, Lum-Jones A, Henderson BE, Kolonel LN, Le Marchand L. Well-done meat consumption, NAT1 and NAT2 acetylator genotypes and prostate cancer risk: the multiethnic cohort study. Cancer Epidemiol Biomarkers Prev. 2010; 19:1866-1870.

27. Iguchi T, Sugita S, Wang CY, Newman NB, Nakatani T, Haas GP. MnSOD genotype and prostate cancer risk as a function of NAT genotype and smoking status. In Vivo. 2009; 23:7-12.

28. Srivastava DS, Mittal RD. Genetic polymorphism of the $\mathrm{N}$-acetyltransferase 2 gene, and susceptibility to prostate cancer: a pilot study in north Indian population. BMC Urol. $2005 ; 5: 12$.
29. Costa S, Pinto D, Morais A, Vasconcelos A, Oliveira J, Lopes C, Medeiros R. Acetylation genotype and the genetic susceptibility to prostate cancer in a southern European population. Prostate. 2005; 64:246-252.

30. Rovito PJ, Morse PD, Spinek K, Newman N, Jones RF, Wang CY, Haas GP. Heterocyclic amines and genotype of $\mathrm{N}$-acetyltransferases as risk factors for prostate cancer. Prostate Cancer Prostatic Dis. 2005; 8:69-74.

31. Gao JP, Huang YD, Yang GZ, Yang YQ. [Relationship between genetic polymorphisms of metabolizing enzymes and prostate cancer]. [Article in Chinese]. Zhonghua Nan Ke Xue. 2003; 9:32-35.

32. Hein DW, Leff MA, Ishibe N, Sinha R, Frazier HA, Doll MA, Xiao GH, Weinrich MC, Caporaso NE. Association of prostate cancer with rapid $\mathrm{N}$-acetyltransferase 1 (NAT $1 * 10)$ in combination with slow $\mathrm{N}$-acetyltransferase 2 acetylator genotypes in a pilot case-control study. Environ Mol Mutagen. 2002; 40:161-167.

33. Wadelius M, Autrup JL, Stubbins MJ, Andersson SO, Johansson JE, Wadelius C, Wolf CR, Autrup H, Rane A. Polymorphisms in NAT2, CYP2D6, CYP2C19 and GSTP1 and their association with prostate cancer. Pharmacogenetics. 1999; 9:333-340.

34. Agundez JA, Martinez C, Olivera M, Gallardo L, Ladero JM, Rosado C, Prados J, Rodriguez-Molina J, Resel L, Benitez J. Expression in human prostate of drug- and carcinogen-metabolizing enzymes: association with prostate cancer risk. Br J Cancer. 1998; 78:1361-1367.

35. Hein DW, Doll MA, Fretland AJ, Leff MA, Webb SJ, Xiao GH, Devanaboyina US, Nangju NA, Feng Y. Molecular genetics and epidemiology of the NAT1 and NAT2 acetylation polymorphisms. Cancer Epidemiol Biomarkers Prev. 2000; 9:29-42.

36. Vatsis KP, Martell KJ, Weber WW. Diverse point mutations in the human gene for polymorphic $\mathrm{N}$-acetyltransferase. Proc Natl Acad Sci U S A. 1991; 88:6333-6337.

37. Hein DW. N-acetyltransferase SNPs: emerging concepts serve as a paradigm for understanding complexities of personalized medicine. Expert Opin Drug Metab Toxicol. 2009; 5:353-366.

38. Bell DA, Badawi AF, Lang NP, Ilett KF, Kadlubar FF, Hirvonen A. Polymorphism in the N-acetyltransferase 1 (NAT1) polyadenylation signal: association of NAT1*10 allele with higher $\mathrm{N}$-acetylation activity in bladder and colon tissue. Cancer Res. 1995; 55:5226-5229.

39. Fukutome K, Watanabe M, Shiraishi T, Murata M, Uemura H, Kubota Y, Kawamura J, Ito H, Yatani R. N-acetyltransferase 1 genetic polymorphism influences the risk of prostate cancer development. Cancer Lett. 1999; 136:83-87.

40. Taylor JA, Umbach DM, Stephens E, Castranio T, Paulson D, Robertson C, Mohler JL, Bell DA. The role of $\mathrm{N}$-acetylation polymorphisms in smoking-associated bladder cancer: evidence of a gene-gene-exposure threeway interaction. Cancer Res. 1998; 58:3603-3610. 
41. Risch A, Wallace DM, Bathers S, Sim E. Slow N-acetylation genotype is a susceptibility factor in occupational and smoking related bladder cancer. Hum Mol Genet. 1995; 4:231-236.

42. Kampman E, Slattery ML, Bigler J, Leppert M, Samowitz W, Caan BJ, Potter JD. Meat consumption, genetic susceptibility, and colon cancer risk: a United States multicenter case-control study. Cancer Epidemiol Biomarkers Prev. 1999; 8:15-24.

43. Munafo MR, Clark TG, Flint J. Assessing publication bias in genetic association studies: evidence from a recent metaanalysis. Psychiatry Res. 2004; 129:39-44.

44. Zhang S, Tang Q, Wu W, Yuan B, Lu C, Xia Y, Ding H, Hu L, Chen D, Sha J, Wang X. Association between DAZL polymorphisms and susceptibility to male infertility: systematic review with meta-analysis and trial sequential analysis. Sci Rep. 2014; 4:4642.

45. Thorlund K, Devereaux PJ, Wetterslev J, Guyatt G, Ioannidis JP, Thabane L, Gluud LL, Als-Nielsen B, Gluud C. Can trial sequential monitoring boundaries reduce spurious inferences from meta-analyses? Int $\mathrm{J}$ Epidemiol. 2009; 38:276-286. 